\title{
The association of XRCCI polymorphism with osteosarcoma risk, clinicopathologic features, and prognosis in a Chinese Han population
}

This article was published in the following Dove Press journal: Cancer Management and Research

\author{
Ying-Guang $\mathrm{Wu}^{\prime}$ \\ Hong-Fu $\mathrm{Li}^{2}$ \\ Yan-Jun Ren' \\ De-Bo Zou' \\ Kai-Ning Zhang' \\ Xing Xiao' \\ 'Department of Spine Surgery, \\ Qianfoshan Hospital, Shandong \\ University, Jinan, Shandong, China; \\ ${ }^{2}$ Department of Orthopedics, \\ Second Hospital of Haibei Tibetan \\ Autonomous Region, Menyuan, \\ Qinghai, China
}

\begin{abstract}
Introduction: The association of single-nucleotide polymorphisms at X-ray repair crosscomplementing group-1 (XRCC1) with osteosarcoma (OS) development has not been fully clear to date. The aim of the present study is to evaluate the association of XRCC1 polymorphisms with risk, clinicopathologic features, and prognosis in Chinese OS patients.

Methods: A total of 146 patients with primary OS and 248 age- and gender-matched controls were included in the present study. The frequencies of four XRCC1 polymorphisms (rs25487, rs1799782, rs25489, and rs3213245) were determined between OS patients and controls. The association of XRCC1 polymorphism with clinicopathologic characteristics, prognosis, and XRCC1 expression was further evaluated.

Results: Compared with TT genotype, individuals carrying the minor C allele (TC+ CC) of rs3213245 had significantly increased risk of OS development ( $\mathrm{OR}=1.83,95 \% \mathrm{CI} 1.14-3.00)$. OS patients carrying TC genotype and C allele at rs3213245 were more likely to be with larger tumor size and metastasis. Survival analysis demonstrated that OS patients carrying $\mathrm{C}$ allele $(\mathrm{TC}+\mathrm{CC})$ at rs3213245 had shorter survival time than those with TT genotype. The T to C substitution at rs3213245 could decrease $X R C C 1$ gene transcriptional activity in vitro. XRCC1 mRNA and protein expression levels were lower in OS patients carrying TC or CC genotype at rs3213245. Besides, no significant association of rs25487, rs1799782, and rs25489 with OS was observed.
\end{abstract}

Conclusion: In conclusion, these findings revealed that XRCC1 rs3213245 polymorphism was associated with increased risk of OS, which could affect XRCC1 expression in vitro and in vivo.

Keywords: osteosarcoma, $\mathrm{XRCC} 1$, polymorphism, prognosis

\section{Introduction}

Osteosarcoma (OS) is the most common primary malignant bone cancer, which mainly affects children, adolescents, and young adults. ${ }^{1}$ Due to the lack of specific presentation and high aggressiveness, almost $20 \%$ of OS patients show clinically detectable metastases at presentation, and most metastases occur in the lung. Currently, for early-stage disease without metastases, treatment strategies mainly include standard chemotherapy administered before and after surgery followed by radiation, with a 5-year survival rate of $60 \%-70 \%$ for OS patients. ${ }^{2}$ However, prognosis of OS patients with locally advanced or distant metastasis when diagnosed or in recurrent disease is still poor, and a previous study demonstrated that the median survival time for these late-stage patients was only 23 months. ${ }^{3}$ Although the response to treatment in a subset of OS patients was improved due to the development of chemotherapy and targeted therapy,
Department of Spine Surgery, Qianfoshan Hospital, Shandong University, Number 16766 Jingshi Road, Jinan, Shandong, 2500 I4, China

Email xxboneoncol@yeah.net 
overall survivals were not significantly prolonged. ${ }^{2,4}$ Therefore, there is an urgent need for comprehensive understanding of the underlying factor of OS biology, which could assist in the identification of more effective molecular biomarkers for diagnosis, prognosis, and development of a more effective treatment strategy and therapeutic target to improve patient survival.

The precise etiology of OS remains partially unknown. However, as a multifactorial disease, several factors have been identified in OS development, including environmental and genetic factors. ${ }^{5}$ Genetic factors might play a key role in OS pathogenesis. To date, several previous studies have reported associations of DNA repair gene variants in biologically plausible pathways with OS risk, ${ }^{6-10}$ such as ERCC1, ERCC2, $A P E$, and so on. X-ray repair cross-complementing group 1 $(X R C C 1)$ is a member of the DNA repair gene family, which is responsible for fixing DNA base damage and single-strand breaks through interacting with DNA components at the damage site. ${ }^{11,12}$ XRCC1 polymorphisms have been linked to cancer development in several types of cancer. ${ }^{13-20}$ However, the association of XRCC1 polymorphism with OS still was not fully clear. Therefore, we conducted the present study to determine the association between rs25487, rs1799782, rs25489, and rs3213245 polymorphisms and OS in a cohort of Chinese Han population.

\section{Materials and methods Study subjects}

In this case-control study, a total of 146 patients with primary OS were enrolled to the Department of Orthopedics of Shandong Qianfoshan Hospital (Jinan, China) between January 2013 and December 2015. All subjects had to meet the following criteria: Chinese Han descent; newly diagnosed, previously untreated, histologically confirmed primary OS; and no history of other cancers. Two hundred and forty-eight age- and gender-matched controls without orthopedic disease or cancer were included. Prior to treatment, peripheral blood samples were collected from each recruited subject for DNA extraction. Demographic and clinicopathologic features of all OS patients were obtained from the medical records, including age, gender, tumor location, tumor size, tumor grade, tumor metastasis, and response to chemotherapy. One hundred and fourteen of 146 OS patients were involved in follow-up analysis. Overall survival was defined as the time from surgery to the date of OS-related death or last follow-up. This study was approved by the Institutional Review Board of Shandong Qianfoshan Hospital. Written informed consent was obtained from all participants. We confirmed that all methods were performed in according with the relevant guidelines and regulation. All identifying information of patient had been removed from all text/figures/tables.

\section{Single-nucleotide polymorphisms (SNP) genotyping analysis}

Genomic DNA from peripheral lymphocytes was obtained using a QIAamp DNA Blood Mini kit (Qiagen NV, Venlo, the Netherlands) based on the manufacturer's instructions. Genotyping analysis was performed using custom TaqMan SNP genotyping assays with TaqMan genotyping master mix (Thermo Fisher, CA, USA) and an ABI Prism 7900HT genetic detection system. The final reactive system is $25 \mu \mathrm{L}$, including $12.50 \mu \mathrm{L}$ of master mix, $1.25 \mu \mathrm{L}$ of assay mix, and $11.25 \mu \mathrm{L}$ of ddH2O. Reactive conditions of TaqMan SNP Genotyping assay were as follows: $95^{\circ} \mathrm{C}$ for 15 seconds and $60^{\circ} \mathrm{C}$ for 1 minute, repeated for 40 cycles. Eventually, the frequencies of genotype or allele of XRCC1 polymorphisms were determined based on the allelic discrimination plots. To ensure the accuracy of the results, $10 \%$ of samples were randomly chosen for repeated assays, and no inconsistent results were found.

\section{Plasmid constructs}

According to the previously described methods, we constructed a luciferase reporter plasmid using the pGL3-Basic reporter vector (Promega, Madison, WI, USA). ${ }^{15}$ A fragment (from -818 to -22 ) in the $\mathrm{XRCC1}$ promoter region from the subjects homozygous for rs3213245 $\mathrm{T}$ or $\mathrm{C}$ allele was amplified using the following primers: $5^{\prime}$-AAACGCGTTTGCGTAGAATCCAGGTTCC-3' and 5'-AAAGATCTTGGCCAGAAGGATGAGGTAG-3' ${ }^{\prime}$. Both the amplified fragments and pGL3-basic vector were digested with Mlu I (A^CGCGT/ $\left.\mathrm{TGCGC}^{\wedge} \mathrm{A}\right)$ and Bgl II (A^GATCT/TCTAG $\left.{ }^{\wedge} \mathrm{A}\right)$ enzymes (New England BioLabs Inc., Ipswich, MA, USA), and the amplified fragments were cloned into pGL3-basic vector. Subsequently, the vectors were sequenced to validate the orientation and integrity of plasmid construct.

\section{Human OS cell culture and luciferase assay}

SaOS-2 and MG-63 cells (purchased from Institute of Cellular Research, Chinese Academy of Science, Shanghai, China) were cultured in RPMI 1640 containing fetal bovine serum (10\%; HyClone, Logan, UT, USA), penicillin (100 units/ mL; Gibco; Thermo Fisher Scientific, Waltham, MA, USA), and streptomycin (100 $\mu \mathrm{g} / \mathrm{mL}$; Gibco). SaOS-2 and MG-63 cells were seeded in 24-well plates before transfection. pGL3 
luciferase reporters containing rs3213245 T or C allele were transfected into cells with the PolyJet DNA In Vitro Transfection Reagent (Signagen Laboratories, MD, USA). After 24 hours of incubation, cells were lysed in passive lysis buffer. Firely and Renilla luciferase signals were measured with a dual-luciferase reporter assay system and a microplate reader. The negative control is the pGL3-basic vector without an insert, while Renilla plasmid (pRL-SV40) was used as an internal control. Luciferase assay was carried out using the Dual Glo Luciferase System (Promega, CA, USA) according to the manufacture's instruction. To ensure the accuracy of experiments, independent triplicate wells were done.

\section{RNA isolation and quantitative real-time PCR (qRT-PCR)}

Total RNA was obtained from tumor tissue. Approximately $1 \mu \mathrm{g}$ of total RNA was used for cDNA synthesis by a reverse transcription kit and qRT-PCR was performed after isolating cDNA. XRCC1 mRNA expression was determined relative to GAPDH using the equation 2$\Delta \Delta \mathrm{Ct}\left(\Delta \mathrm{Ct}=\mathrm{Ct}_{\mathrm{XRCC} 1}-\mathrm{Ct}_{\mathrm{GAPDH}}\right.$. The primers were as follows: 5'-AAGCAACCCCAGACCAAAAC-3' (forward) and 5'-CCCCATTGTCCTGTCCTTCT-3' (reverse) for XRCC1, and 5'-CCAGAACATCATCCCTGCCT-3' (forward) and 5'-CCTGCTTCACCACCTTCTTG-3' (reverse) for GAPDH.

\section{Protein isolation and Western blotting}

The 146 OS patients were divided into three groups based on the distribution of rs3213245 genotype. To assess the effect of rs3213245 polymorphism on XRCC1 protein expression, we randomly selected 12 OS tumor tissues and isolated the protein according to the previously described method. ${ }^{10}$ Subsequently, Western blotting was performed to evaluate the XRCC1 protein expression. Thirty microgram of extracted protein was subjected to $10 \%$ SDS-PAGE and transferred to PVDF membranes (EMD Millipore, Billerica, MA, USA). Membranes were blocked with 5\% nonfat milk in TBST buffer (containing $0.1 \%$ Tween-20) and then incubated with human XRCC1 antibody (1:1,000 dilution, ab1838, Mouse IgG; Abcam, Cambridge, UK) and GAPDH antibody (1:1,000 dilution, sc-32233; Mouse IgG; Santa Cruz Biotechnology Inc., Dallas, TX, USA) at $4^{\circ} \mathrm{C}$ overnight. Horseradish peroxidase (HRP)-conjugated antirabbit IgG and antimouse IgG were used as the secondary antibody (1:5,000 dilution, Beoytime Biotech, Shanghai, China). Signals were captured using an HRP chemiluminescent kit (Beoytime Biotech).

\section{Statistical analysis}

The association between XRCC1 genotypes and OS risk was assessed with ORs using an unconditional logistic regression model. Deviations from Hardy-Weinberg equilibrium were assessed with a chi-squared test. Survival probabilities were determined with a Kaplan-Meier curve, and significant differences were analyzed with a logrank test. Cox proportional hazards models were used to analyze the associations between genotypes and OS survival. HR and 95\% CIs were estimated using multivariable models. XRCC1 expression among patients with different genotypes were compared with a Student's $t$-test. All statistical analyses were performed with SPSS 22.0 (SPSS Inc., Chicago, IL, USA) and GraphPad Prism 7.0 (GraphPad Software, Inc., La Jolla, CA, USA). $P<0.05$ was considered statistically significant.

\section{Results \\ Association of $\mathrm{XRCCI}$ polymorphisms with OS risk}

The clinical characteristics of OS patients and normal controls were present in Table 1. No significant difference in age and gender could be observed. All the genotype frequencies of rs25487, rs1799782, rs25489, and rs3213245 did not deviate from Hardy-Weinberg equilibrium in control groups (all $P>0.10$ ). Significant differences between OS patients and controls could be only observed in rs3213245 (Table 2). Under the additive model, significantly increased risk of OS could be observed in subjects with TC genotype when compared with TT genotype at rs3213245 (OR =1.74, 95\% CI 1.07-2.84). Individuals with the minor $\mathrm{C}$ allele (TC+ $\mathrm{CC})$ showed a significant increase in the risk of OS development (OR $=1.83,95 \%$ CI 1.14-3.00). Similarly, individuals carrying the $\mathrm{C}$ allele had significantly increased risk of developing OS compared with $\mathrm{T}$ allele $(\mathrm{OR}=1.79,95 \% \mathrm{CI}$ 1.16-2.76). For rs25487, patients with the minor A allele seem to have a lower risk of OS development compared with $\mathrm{G}$ allele $(\mathrm{OR}=0.78,95 \%$ CI $0.57-1.08)$, but without significant difference. Under dominant, recessive, and additive models, a decreased risk of OS development could be observed in subjects carrying the A allele, but the difference did not reach statistical significance (all $P>0.05$ ). In addition, the distribution and frequency of rs1799782 and rs25489 were similar between OS patients and normal controls. Furthermore, Haploview analysis did not reveal an LD among these four XRCC1 polymorphism in our cohort. Therefore, the association of the XRCC1 haplotype with OS risk was not further evaluated. 
Table I The characteristics of OS patients and normal controls

\begin{tabular}{|l|l|l|l|}
\hline Characteristics & $\begin{array}{l}\text { OS cases } \\
(\mathbf{n}=\mathbf{1 4 6})\end{array}$ & $\begin{array}{l}\text { Normal } \\
\text { controls } \\
\text { (n=248) }\end{array}$ & P-value \\
\hline Age (years) & 85 & 147 & 0.92 \\
$<20$ & 61 & 101 & \\
$>20$ & 86 & 159 & 0.33 \\
Gender (n, \%) & 60 & 89 & \\
Male & & & \\
Female & 83 & & \\
Tumor location & 42 & & \\
Femur & 21 & & \\
Tibia & & \\
Other & 76 & & \\
Tumor size (cm) & 70 & & \\
$<6$ & & & \\
$>6$ & 71 & & \\
Tumor grade & 75 & & \\
Low & & \\
High & 46 & \\
Clinical stage & 73 & \\
IIA & 27 & \\
IIB & 100 & \\
III & 46 & \\
Distant metastasis & 81 & \\
Negative & 65 & \\
Positive & & \\
Response to chemotherapy & & \\
Poor & & \\
Good & & \\
\hline Abrevion & & \\
& & \\
\end{tabular}

rs32 I 3245 and clinicopathologic characteristics

The results of stratification analysis by clinicopathologic characteristics were described in Table 3. OS patients carrying TC genotype at rs3213245 were more likely to be with larger tumor size (OR $=2.21,95 \%$ CI 1.04-4.72) and metastasis ( $\mathrm{OR}=2.55,95 \% \mathrm{CI} 1.18-5.51)$. Patients carrying $\mathrm{C}$ allele at rs3213245 were more susceptible to be with metastases at diagnosis (OR $=2.86,95 \%$ CI $1.50-5.43$ ). For response to chemotherapy, OS patients carrying TC genotype or $\mathrm{C}$ allele tended to have a good response to chemotherapy, but without significant difference $(P=0.09, P=0.35)$. In addition, we could observe that all three OS patients with CC genotype were in the femur/tibia/fibula, had metastases, and showed good response to chemotherapy. Besides, no other significant relationship could be observed.

\section{rs32I 3245 and prognosis}

Kaplan-Meier curves were plotted to determine the association of survival rate with rs3213245. Significant difference in survival rate was detected among patients with different genotypes at rs3213245 (Figure 1). The results revealed that OS patients carrying TC + CC genotype at rs3213245 had shorter survival time than those with TT genotype alone

Abbreviation: OS, osteosarcoma.

Table 2 Genotype frequencies of XRCCI polymorphisms among OS patients and normal controls

\begin{tabular}{|c|c|c|c|c|c|c|}
\hline SNP & Genotype/allele & OS (n, \%) & Controls (n, \%) & HWE & OR (95\% Cl) & $P$-value \\
\hline \multirow[t]{6}{*}{ rs3213245 } & $\mathrm{TT}$ & $103(70.5)$ & $202(81.5)$ & 0.36 & Reference & \\
\hline & TC & $40(27.4)$ & $45(18.1)$ & & $1.74(1.07-2.84)$ & 0.03 \\
\hline & $\mathrm{CC}$ & $3(2.1)$ & I (0.4) & & $5.88(0.60-57.5)$ & 0.13 \\
\hline & $\mathrm{TC}+\mathrm{CC}$ & $43(29.5)$ & $46(18.5)$ & & $1.83(1.14-3.00)$ & 0.02 \\
\hline & $\mathrm{T}$ & $246(84.2)$ & $449(90.5)$ & & Reference & \\
\hline & C & $46(15.8)$ & $47(9.5)$ & & $1.79(1.16-2.76)$ & 0.01 \\
\hline \multirow[t]{6}{*}{ rsl799782 } & $\mathrm{CC}$ & $76(52.1)$ & $130(52.4)$ & 0.84 & Reference & \\
\hline & CT & $60(4 I . I)$ & $100(40.3)$ & & I.03 (0.67-I.57) & 0.91 \\
\hline & $\mathrm{TT}$ & $10(6.8)$ & $18(7.3)$ & & $0.95(0.42-2.17)$ & 1.00 \\
\hline & $\mathrm{CT}+\mathrm{TT}$ & $70(47.9)$ & $118(47.6)$ & & $1.02(0.67-1.53)$ & 0.94 \\
\hline & $\mathrm{C}$ & $212(72.6)$ & $360(72.6)$ & & Reference & \\
\hline & $\mathrm{T}$ & $80(27.4)$ & $136(27.4)$ & & $1.00(0.72-1.38)$ & 0.90 \\
\hline \multirow[t]{6}{*}{ rs25489 } & GG & $119(81.5)$ & $208(83.9)$ & 0.85 & Reference & \\
\hline & GA & $26(17.8)$ & $38(I 5.3)$ & & $1.20(0.69-2.07)$ & 0.57 \\
\hline & AA & I $(0.7)$ & $2(0.8)$ & & $0.87(0.08-9.74)$ & 0.91 \\
\hline & $\mathrm{GA}+\mathrm{AA}$ & $27(18.5)$ & $40(16.1)$ & & $1.18(0.69-2.02)$ & 0.58 \\
\hline & G & $264(90.4)$ & 454 (9I.5) & & Reference & \\
\hline & $A$ & $28(9.6)$ & $42(8.5)$ & & I.II (0.69-1.89) & 0.61 \\
\hline \multirow[t]{6}{*}{ rs25487 } & GG & $83(56.8)$ & $126(50.8)$ & 0.66 & Reference & \\
\hline & $\mathrm{GA}$ & $53(36.3)$ & $103(4 \mid .5)$ & & $0.78(0.5 \mathrm{I}-1.20)$ & 0.28 \\
\hline & $A A$ & $10(6.9)$ & $24(9.7)$ & & $0.63(0.29-1.39)$ & 0.34 \\
\hline & $\mathrm{GA}+\mathrm{AA}$ & $63(43.2)$ & $127(5 \mid .2)$ & & $0.75(0.50-1.14)$ & 0.18 \\
\hline & G & $219(75.0)$ & $355(71.6)$ & & Reference & \\
\hline & $A$ & $73(25.0)$ & $15 \mid(30.4)$ & & $0.78(0.57-1.08)$ & 0.16 \\
\hline
\end{tabular}

Note: Statistically significant genotypes/alleles and values are shown in bold.

Abbreviations: HWE, Hardy Weinberg Equilibrium; OS, osteosarcoma; SNP, single-nucleotide polymorphism; XRCCI, X-ray repair cross-complementing group-I. 
Table 3 The association of rs32 13245 with clinicopathologic characteristics in OS patients

\begin{tabular}{|c|c|c|c|c|c|c|}
\hline \multirow[t]{2}{*}{ Characteristic } & \multirow[b]{2}{*}{ Patients (N) } & \multicolumn{3}{|c|}{ Genotypes } & \multicolumn{2}{|l|}{ Allele } \\
\hline & & TT & TC & $\mathrm{CC}$ & $\mathbf{T}$ & $\mathbf{C}$ \\
\hline \multicolumn{7}{|l|}{ Age (years) } \\
\hline$<20$ & 85 & 59 & 25 & 1 & 143 & 27 \\
\hline$>20$ & 61 & 44 & 15 & 2 & 103 & 19 \\
\hline OR $(95 \% \mathrm{Cl})$ & & Reference & $0.81(0.38-1.70)$ & $2.68(0.24-30.5)$ & Reference & $0.98(0.52-1.85)$ \\
\hline$P$-value & & - & 0.71 & 0.58 & - & 0.94 \\
\hline \multicolumn{7}{|l|}{ Gender (n, \%) } \\
\hline Male & 86 & 65 & 20 & I & 150 & 22 \\
\hline Female & 60 & 38 & 20 & 2 & 96 & 24 \\
\hline OR $(95 \% \mathrm{Cl})$ & & Reference & I.7। (0.82-3.58) & $3.42(0.30-39.0)$ & Reference & $1.7 \mid(0.9 \mid-3.21)$ \\
\hline$P$-value & & - & 0.19 & 0.56 & - & 0.11 \\
\hline \multicolumn{7}{|l|}{ Tumor location } \\
\hline Femur/Tibia/Fibula & 125 & 82 & 30 & 3 & 194 & 36 \\
\hline Other & 21 & 11 & 10 & 0 & 32 & 10 \\
\hline OR $(95 \% \mathrm{Cl})$ & & Reference & $2.49(0.96-6.45)$ & - & Reference & $1.68(0.76-3.73)$ \\
\hline$P$-value & & - & 0.07 & - & - & 0.26 \\
\hline \multicolumn{7}{|l|}{ Tumor size (cm) } \\
\hline$<6$ & 71 & 56 & 14 & 1 & 126 & 16 \\
\hline$>6$ & 75 & 47 & 26 & 2 & 120 & 30 \\
\hline OR $(95 \% \mathrm{Cl})$ & & Reference & $2.21(1.04-4.72)$ & $2.38(0.2 \mathrm{I}-27.1)$ & Reference & $1.97(1.02-3.80)$ \\
\hline$P$-value & & - & 0.35 & 0.60 & - & 0.06 \\
\hline \multicolumn{7}{|c|}{ Pathological grade } \\
\hline High differentiation & 76 & 57 & 18 & 1 & 132 & 20 \\
\hline Low differentiation & 70 & 46 & 22 & 2 & 114 & 26 \\
\hline OR $(95 \% \mathrm{Cl})$ & & Reference & $1.51(0.73-3.16)$ & $2.48(0.22-28.1)$ & Reference & $1.5 \mathrm{I}(0.80-2.84)$ \\
\hline$P$-value & & - & 0.35 & 0.59 & - & 0.26 \\
\hline \multicolumn{7}{|c|}{ Metastasis at diagnosis } \\
\hline Negative & 100 & 78 & 22 & 0 & 178 & 22 \\
\hline Positive & 46 & 25 & 18 & 3 & 68 & 24 \\
\hline OR $(95 \% \mathrm{Cl})$ & & Reference & $2.55(1.18-5.5 I)$ & - & Reference & $2.86(1.50-5.43)$ \\
\hline$P$-value & & - & 0.02 & 0.01 & - & $<0.01$ \\
\hline \multicolumn{7}{|c|}{ Response to chemotherapy } \\
\hline Poor & 81 & 61 & 20 & 0 & 142 & 20 \\
\hline Good & 65 & 42 & 20 & 3 & 104 & 26 \\
\hline OR $(95 \% \mathrm{Cl})$ & & Reference & $1.45(0.70-3.03)$ & - & Reference & $1.78(0.94-3.35)$ \\
\hline$P$-value & & - & 0.35 & - & - & 0.09 \\
\hline
\end{tabular}

Note: Statistically significant values are shown in bold.

Abbreviations: -, not applicable; OS, osteosarcoma.

(Figure 1, $P=0.04$ ). The multivariate analysis of survival time using the Cox proportional hazards model revealed that distant metastases and response to chemotherapy were two independent prognostic factors for worse survival in OS patients ( $\mathrm{HR}=4.01,95 \%$ CI $2.34-6.09 ; \mathrm{HR}=2.32,95 \% \mathrm{CI}$ 1.67-4.57; Table 4). However, rs3213245 was not independently associated with overall survival ( $\mathrm{HR}=1.41,95 \% \mathrm{CI}$ $0.86-2.62$ ).

\section{rs32I3245 and XRCCI transcriptional activity, $\mathrm{XRCCl}$ expression}

Compared with $\mathrm{T}$ allele, the constructed luciferase reporter vectors with $\mathrm{C}$ allele could yield a $60 \%$ decrease in the relative luciferase activities in SaOS-2 and MG-63 cells (Figure 2). We further evaluate the expression levels of $\mathrm{XRCC} 1$ in OS patients to determine the relationship between rs3213245 polymorphism and XRCC1 expression. Similar to transcriptional activity, XRCC1 mRNA levels were significantly decreased in OS patients carrying the $\mathrm{C}$ allele (Figure $3 \mathrm{~A}, P<0.01$ ). The differences between $\mathrm{CC}$ and TC genotypes were not statistically significant due to a smaller sample size. In addition, XRCC1 protein expression levels of patients carrying $\mathrm{TC}$ or $\mathrm{CC}$ genotype were lower than those with TT genotype (Figure 3B). Taken together, the observation of lower XRCC1 expression in OS patients carrying $\mathrm{TC}$ or CC genotype suggested that $\mathrm{C}$ allele at rs3213245 is a genetic risk factor for OS. 


\section{Discussion}

$\mathrm{XRCC} 1$ is involved in base excision repair (BER) of DNA and has variant genotypes associated with modified DNA repair function. In XRCC1 knockout mice, spontaneous chromosome aberrations and embryonic lethality supported the importance of XRCC1 in genome maintenance. ${ }^{21}$ In the present study, we assessed the association of four common XRCC1 gene polymorphisms with OS, and observed that $\mathrm{C}$ allele at rs3213245 was associated with increased risk of OS development, larger tumor size as well as metastases compared with T allele and the homozygous TT genotype. Survival analysis showed that patients carrying $\mathrm{C}$ allele at rs3213245 had worse survival. In addition, the present study also found that the $\mathrm{T}$ to $\mathrm{C}$ substitution at rs3213245 could decrease the transcription activity of XRCC1 gene in vitro. Furthermore, XRCC1 mRNA and protein expression were

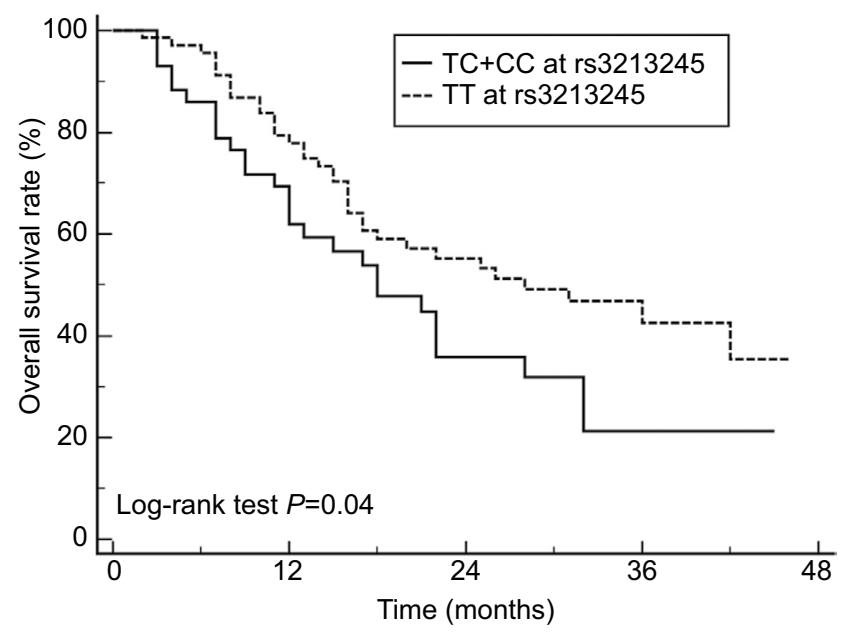

Figure I Kaplan-Meier survival curves.

Notes: Kaplan-Meier survival curves of OS patients with different rs3213245 genotypes (28 events for TC + CC, 35 events for TT), XRCCI rs3213245 polymorphism was correlated with the overall survival in OS patients.

Abbreviations: OS, osteosarcoma; $\mathrm{XRCCI}, \mathrm{X}$-ray repair cross-complementing group-I. downregulated in vivo in OS patients carrying the $\mathrm{C}$ allele, suggesting that rs3213245 is indeed a functional polymorphism both in vitro and in vivo as well as being a genetic biomarker for the risk and prognosis of OS.

DNA repair systems play a vital role in protecting against tumorigenesis, and genetic defects in DNA repair systems could cause tumors. ${ }^{22}$ Accumulated evidence revealed that the polymorphisms located in the coding region could reduce the DNA repair capacity, and the genetic polymorphism in the regulatory region could decrease the expression of DNA repair genes, which were linked to the risk of tumor development, such as APE1, BRCA1, XPF, and ERCC1. ${ }^{10,23-26}$ The BER pathway is one important mechanism of all DNA repair pathways, which could repair DNA base damage and single-strand breaks. As a scaffold protein in BER, XRCC1 assembles a DNA protein complex at the damaged site, while

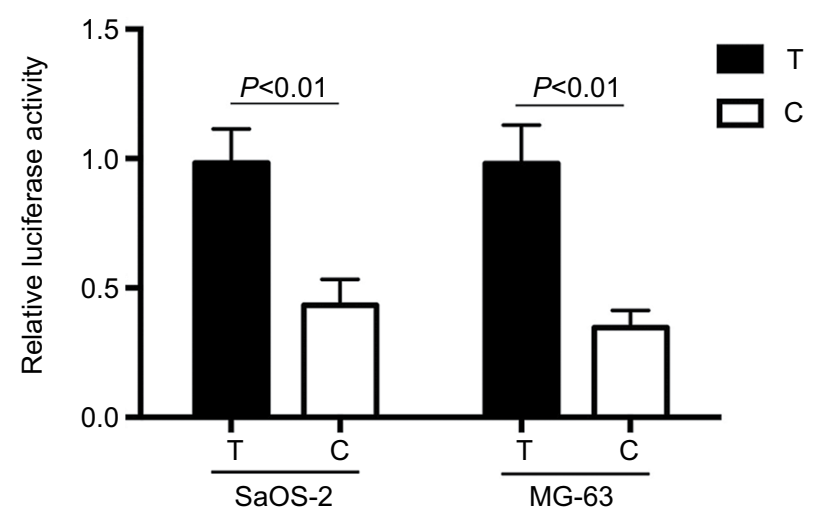

Figure 2 Effect of rs32/3245 polymorphism in the XRCCI transcriptional activity. Notes: Schematic representation of reporter plasmids containing $\mathrm{T}$ or $\mathrm{C}$ allele at rs3213245, which was inserted into upstream of the luciferase reporter gene in the pGL3 plasmid. pRL-SV40 were cotransfected into SaOS2, MG-63 cells as the internal control of Renilla luciferase. Columns, mean from three independent experiments; bars, SD.

Abbreviation: $\mathrm{XRCCI}, \mathrm{X}$-ray repair cross-complementing group-I.

Table 4 Univariate and multivariate Cox proportional hazard analyses for OS patients' overall survival

\begin{tabular}{|c|c|c|c|}
\hline \multirow[t]{2}{*}{ Factor } & \multirow[t]{2}{*}{ Category } & Univariate & Multivariate \\
\hline & & HR (95\% CI) & HR (95\% CI) \\
\hline Age (years) & $\geq 20 /<20$ & $1.15(0.67-2.06)$ & \\
\hline Gender & Male/female & $1.4 \mid(0.80-2.97)$ & \\
\hline Tumor location & Femur + tibia/other & $0.91(0.57-2.03)$ & \\
\hline Tumor size & $\geq 6 /<6 \mathrm{~cm}$ & $2.05(0.93-2.78)$ & \\
\hline Tumor grade & Poor/good & $2.11(1.65-3.77)$ & $\mathrm{I} .83(0.66-3.2 \mathrm{I})$ \\
\hline Distant metastases & Yes/no & $4.59(3.66-6.28)$ & $4.01(2.34-6.09)$ \\
\hline Chemotherapy response & Poor/good & $3.70(2.5 I-6.08)$ & $2.32(1.67-4.57)$ \\
\hline rs3213245 & $\mathrm{TC}+\mathrm{CC} / \mathrm{TT}$ & $1.89(1.16-3.07)$ & $1.41(0.86-2.62)$ \\
\hline
\end{tabular}

Note: Statistically significant values are shown in bold.

Abbreviation: OS, osteosarcoma. 


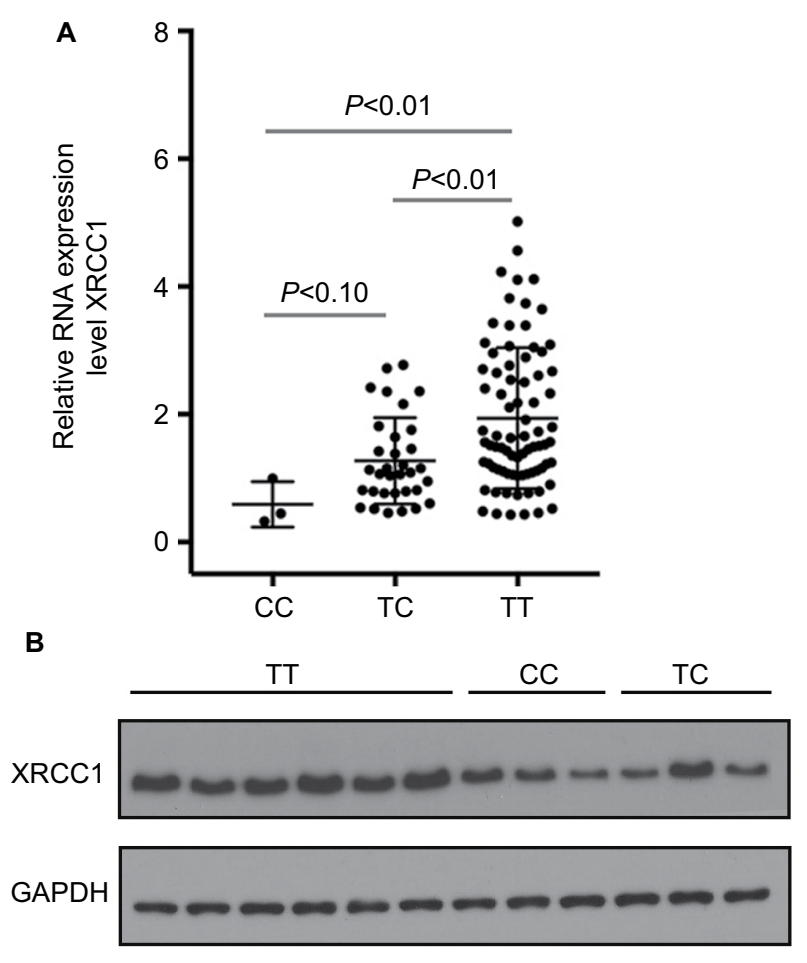

Figure 3 Effect of rs 3213245 polymorphism on XRCCI expression in OS patients. Notes: $(A) X R C C I$ mRNA expression in OS tumor tissues was detected by qRTPCR. (B) XRCCI protein levels were evaluated by Western blotting; OS patients with TT genotype $(n=6)$; OS patients with CC genotype $(n=3)$; OS patients with TC genotype $(n=3)$.

Abbreviations: OS, osteosarcoma; qRT-PCR; quantitative real-time PCR; $X R C C I$, $\mathrm{X}$-ray repair cross-complementing group-I.

interacting with most of the core components that participate in the BER pathway to accomplish DNA repair process. ${ }^{27}$ The XRCC1 gene located on chromosome 19q13.2 consists of 17 exons, and there are several gene polymorphisms within XRCC1 gene. $^{28}$

The most widely studied SNPs were rs1799782, rs25487, and rs25489. rs 1799782 polymorphism is located in exon 6 of XRCC1 and is a C>T substitution in codon 194, which could cause an Arg194Trp variant. Although rs1799782 polymorphism has been extensively studied, contradictory results on its functional significance have been reported. Some previous studies have described a lower DNA repair efficiency associated with the 194Trp variant of XRCC1 protein, ${ }^{29,30}$ while others have reported a decreased DNA repair in 194Arg variant. ${ }^{31-33}$ Also, some investigators have not found any association between rs 1799782 and defective DNA repair. ${ }^{34,35}$ The association of rs 1799782 with cancer risk remains controversial. Some previous studies have reported a significant association of rs 1799782 with thyroid cancer, ${ }^{16}$ colorectal cancer, ${ }^{19}$ lung cancer, ${ }^{17}$ and cervical cancer, ${ }^{18}$ while no significant association could be observed with breast cancer, ${ }^{36}$ pancreatic cancer, ${ }^{37}$ and gastric cancer. ${ }^{15}$ rs 25487 and rs25489 polymorphisms are another widely studied polymorphism with evidences supporting a quantitative effect of the genotype on the phenotype ${ }^{36}$ The homozygous variant $(\mathrm{Gln} / \mathrm{Gln})$ at $\mathrm{rs} 25487$ has been shown to have three to fourfold lower capacity to remove DNA adducts or oxidized DNA damage. Similar to rs 1799782, rs25489 also was shown to have the ability of diminishing the repair function but additional quantitative information was uncertain. The association of rs 25487 and rs 25489 with cancer risk has also remained inconsistent. Several previous studies reported a positive association, while some did not observe the different distribution of these two polymorphisms between cancer patients and normal controls. ${ }^{15,36}$ As for OS, Wang and Wu have reported that rs 25487 polymorphism was significantly associated with the development of OS rather than rs25489. ${ }^{38}$ In the present study, we did not find any significant association between those three polymorphism and OS risk. The possibilities for this discrepancy were different tumor types and different ethnicities.

rs3213245 (-77T $>C)$ is a novel polymorphism located in the promoter-binding sequence. Previous studies have demonstrated that this polymorphism could decrease XRCC1 levels by modifying gene expression. ${ }^{14,15}$ In Chinese population, some investigations have demonstrated that this polymorphism was associated with a higher risk of lung cancer and gastric cancer. Consistent with those results, we also found a positive relationship between rs 3213245 and increased risk of OS. Meanwhile, our results also showed that patients who carried rs3213245 mutation genotype had worse survival. On the basis of the luciferase experiment, this polymorphism could decrease the transcriptional activity of $X R C C 1$ gene. Combined with the phenomenon that OS patients with $\mathrm{C}$ allele had low expression of XRCC1 in OS tumor tissues, these results suggested that rs3213245 polymorphism could be involved in the decreased level of XRCC1 in OS tumor tissue. The accurate mechanism underlying the association of rs3213245 with OS remained unclear, but we speculated this polymorphism may affect susceptibility to OS through the influence of rs3213245 on XRCC1 expression. Previous study also revealed that rs $25487 \mathrm{GG}$ genotype could decrease the function of XRCC1, and those with rs25487 GG genotype showed significantly better ORR; thus, this genotype could act as predictive factors in NSCLC patients treated with platinum-based chemotherapy. ${ }^{39}$ Therefore, in our paper, we observed that patients with rs3213245 minor C allele tended to have good response to chemotherapy, although there are no significant differences. 
Based on genotype effect on phenotype and allele frequency, rs25487 and rs3213245 polymorphism have the largest potential to affect the function of XRCC1, eventually influencing the toxicodynamic response to environmental factors and contributing to the initiation of cancer. Consistent with this theory, our result found a relatively big difference in the frequency and distribution of both rs25487 and rs3213245, although there was no statistical significance in rs25487. In addition, both Hao's and Liu's studies found that rs1799782, rs25487, rs25489, and rs3213245 were in linkage disequilibrium, but we did not observe that rs1799782, rs25487, rs25489, and rs3213245 were in linkage disequilibrium in our study population. . $^{14,15}$ Both studies were performed in the Chinese population, and the frequency of XRCC1 polymorphisms was similar in normal controls among these studies. Therefore, we speculated that one possibility for this discrepancy is the sample size. Hao's and Liu's studies included 2,140 subjects and 1,999 subjects, respectively, but our study only included 146 OS patients and 248 healthy controls. Therefore, whether four SNPs were in linkage disequilibrium, as well as the association of the haplotype of these four SNPs with OS risk, requires future exploration. In addition, the discrepancy in the genetic polymorphism may be attributed to different ethnicities/races. In our study, we only included the Chinese population. The role of these polymorphisms in other ethnic populations should be explored in future research.

Of note, there were several limitations in the present study. First, the sample size was relatively small. In particular, the specific role of rs3213245 and rs25489 in OS development requires further confirmation in a large cohort study because of the low frequency of mutation. Second, although we found that rs 3213245 could affect the transcriptional activity, mRNA and protein expression of XRCC1, the specific mechanism was not clear. Whether this polymorphism could affect the binding affinity of the transcription factor or miRNA also need to be validated in the future. Third, reports on the association of XRCC1 polymorphism with OS were relatively scarce, and we only conducted this study in the Chinese Han population, so the frequency of XRCC1 polymorphisms in other ethnic groups required to be confirmed.

\section{Conclusion}

The present study aimed to determine the association of the XRCC1 polymorphism with OS. The results showed that rs3213245 was associated with the increased risk of OS development. The $\mathrm{C}$ allele at rs 3213245 could reflect a worse prognosis of the OS patients. In addition, the $\mathrm{C}$ allele at rs3213245 could affect XRCC1 production in vitro and in vivo in OS patients.

\section{Acknowledgment}

We thank American Journal Experts for their linguistic assistance during the preparation of this manuscript.

\section{Disclosure}

The authors report no conflicts of interest in this work.

\section{References}

1. Moore DD, Luu HH. Osteosarcoma. Cancer Treat Res. 2014;162:65-92.

2. Sampson VB, Yoo S, Kumar A, Vetter NS, Kolb EA. MicroRNAs and potential targets in osteosarcoma: review. Front Pediatr. 2015;3:69.

3. Fagioli F, Aglietta M, Tienghi A, et al. High-dose chemotherapy in the treatment of relapsed osteosarcoma: an Italian sarcoma group study. $J$ Clin Oncol. 2002;20(8):2150-2156.

4. Xiao X, Wang W, Wang Z. The role of chemotherapy for metastatic, relapsed and refractory osteosarcoma. Paediatr Drugs. 2014;16(6): 503-512.

5. Jaffe N. Osteosarcoma: review of the past, impact on the future. The American experience. Cancer Treat Res. 2009;152:239-262.

6. Cao ZH, Yin HP, Jiang N, Yu B. Association between ERCC1 and ERCC2 gene polymorphisms and chemotherapy response and overall survival in osteosarcoma. Genet Mol Res. 2015;14(3):10145-10151.

7. Biason $P$, Hattinger CM, Innocenti F, et al. Nucleotide excision repair gene variants and association with survival in osteosarcoma patients treated with neoadjuvant chemotherapy. Pharmacogenomics J. 2012;12(6):476-483.

8. Goričar K, Kovač V, Jazbec J, Zakotnik B, Lamovec J, Dolžan V. Genetic variability of DNA repair mechanisms and glutathione-S-transferase genes influences treatment outcome in osteosarcoma. Cancer Epidemiol. 2015;39(2):182-188.

9. Kuo IC, Kao HK, Huang Y, et al. Endoplasmic reticulum aminopeptidase 2 involvement in metastasis of oral cavity squamous cell carcinoma discovered by proteome profiling of primary cancer cells. Oncotarget. 2017;8(37):61698-61708.

10. Xiao X, Yang Y, Ren Y, Zou D, Zhang K, Wu Y. rs1760944 Polymorphism in the APE1 region is associated with risk and prognosis of osteosarcoma in the Chinese Han population. Sci Rep. 2017;7(1):9331.

11. Hanssen-Bauer A, Solvang-Garten K, Akbari M, Otterlei M. X-ray repair cross complementing protein 1 in base excision repair. Int J Mol Sci. 2012;13(12):17210-17229.

12. W D, Qh Z, Zy Z, Zp L, Mr K. Prognostic significance of DNA damage and repair gene expression in human osteosarcoma. Acta Academiae Medicinae Militaris Tertias. 2005;27(13):1370-1373.

13. Findlay JM, Middleton MR, Tomlinson I. A systematic review and meta-analysis of somatic and germline DNA sequence biomarkers of esophageal cancer survival, therapy response and stage. Ann Oncol. 2015;26(4):624-644.

14. Hao B, Miao X, Li Y, et al. A novel T-77C polymorphism in DNA repair gene $\mathrm{XRCC} 1$ contributes to diminished promoter activity and increased risk of non-small cell lung cancer. Oncogene. 2006;25(25): 3613-3620.

15. Liu L, Yuan P, Liu L, et al. A functional $-77 \mathrm{~T}>\mathrm{C}$ polymorphism in $\mathrm{XRCC} 1$ is associated with risk of breast cancer. Breast Cancer Res Treat. 2011;125(2):479-487. 
16. Bashir K, Sarwar R, Fatima S, Saeed S, Mahjabeen I, Akhtar Kayani M. Haplotype analysis of XRCC1 gene polymorphisms and the risk of thyroid carcinoma. J Buon. 2018;23(1):234-243.

17. Jiang $J$, Liang $X$, Zhou $X$, et al. DNA repair gene $X$-ray repair cross complementing group 1 Arg194Trp polymorphism on the risk of lung cancer: a meta-analysis on 22 studies. J Thorac Oncol. 2010;5(11):1741-1747.

18. Li Y, Liu F, Tan SQ, Wang Y, Li SW, Sw L. X-ray repair cross-complementing group 1 (XRCC1) genetic polymorphisms and cervical cancer risk: a huge systematic review and meta-analysis. PLoS One. 2012;7(9):e44441.

19. Wang L, Qian J, Ying C, Zhuang Y, Shang X, Xu F. X-ray cross-complementing groups 1 rs1799782 $\mathrm{C}>\mathrm{T}$ polymorphisms and colorectal cancer susceptibility: A meta-analysis based on Chinese Han population. J Cancer Res Ther. 2016;12(Supplement):C264-C267.

20. Jalali C, Ghaderi B, Amini S, Abdi M, Roshani D. Association of XRCC1 Trp194 allele with risk of breast cancer, and Ki67 protein status in breast tumor tissues. Saudi Med J. 2016;37(6):624-630.

21. Tebbs RS, Flannery ML, Meneses JJ, et al. Requirement for the Xrcc1 DNA base excision repair gene during early mouse development. Dev Biol. 1999;208(2):513-529.

22. Misteli T, Soutoglou E. The emerging role of nuclear architecture in DNA repair and genome maintenance. Nat Rev Mol Cell Biol. 2009;10(4):243-254.

23. Yang M, Guo H, Wu C, et al. Functional FEN1 polymorphisms are associated with DNA damage levels and lung cancer risk. Hum Mutat. 2009;30(9):1320-1328.

24. Yu D, Zhang X, Liu J, et al. Characterization of functional excision repair cross-complementation group 1 variants and their association with lung cancer risk and prognosis. Clin Cancer Res. 2008;14(9):2878-2886.

25. Chan KY, Liu W, Long JR, et al. Functional polymorphisms in the BRCA1 promoter influence transcription and are associated with decreased risk for breast cancer in Chinese women. J Med Genet. 2009;46(1):32-39.

26. Wang M, Wang M, Yuan L, et al. A novel XPF $-357 \mathrm{~A}>\mathrm{C}$ polymorphism predicts risk and recurrence of bladder cancer. Oncogene. 2010;29(13):1920-1928.

27. Limpose KL, Corbett AH, Doetsch PW. BERing the burden of damage: pathway crosstalk and posttranslational modification of base excision repair proteins regulate DNA damage management. DNA Repair. 2017;56: $51-64$.
28. Singh A, Singh N, Behera D, Sharma S. Association and multiple interaction analysis among five XRCC1 polymorphic variants in modulating lung cancer risk in North Indian population. DNA Repair. 2016;47:30-41.

29. Khosa T, Aslam S, Mustafa S, Akbar A, Shaikh RS, Iqbal F. Association of single nucleotide polymorphisms in XRCC1 (194) and XPD (751) with age-related cataract. Int Ophthalmol. 2018;38(3):1135-1146.

30. Zhou C, Zhou Y, Li J, et al. The Arg194Trp polymorphism in the X-ray repair cross-complementing group 1 gene as a potential risk factor of oral cancer: a meta-analysis. Tohoku J Exp Med. 2009;219(1):43-51.

31. Ginsberg G, Angle K, Guyton K, Sonawane B. Polymorphism in the DNA repair enzyme XRCC1: utility of current database and implications for human health risk assessment. Mutat Res. 2011;727(1-2):1-15.

32. Kang SY, Lee KG, Lee W, et al. Polymorphisms in the DNA repair gene XRCC1 associated with basal cell carcinoma and squamous cell carcinoma of the skin in a Korean population. Cancer Sci. 2007;98(5):716-720.

33. Patel AV, Calle EE, Pavluck AL, Feigelson HS, Thun MJ, Rodriguez C. A prospective study of XRCC1 (X-ray cross-complementing group 1) polymorphisms and breast cancer risk. Breast Cancer Res. 2005;7(6):R1168-1173.

34. Lunn RM, Langlois RG, Hsieh LL, Thompson CL, Bell DA. XRCC1 polymorphisms: effects on aflatoxin B1-DNA adducts and glycophorin A variant frequency. Cancer Res. 1999;59(11):2557-2561.

35. Xue H, Ni P, Lin B, Xu H, Huang G. X-ray repair cross-complementing group 1 (XRCC1) genetic polymorphisms and gastric cancer risk: a HuGE review and meta-analysis. Am J Epidemiol. 2011;173(4):363-375.

36. Qiao L, Feng X, Wang G, Zhou B, Yang Y, Li M. Polymorphisms in BER genes and risk of breast cancer: evidences from 69 studies with 33760 cases and 33252 controls. Oncotarget. 2018;9(22):16220-16233.

37. Chen J, Wang H, Li Z. Association between polymorphisms of X-ray repair cross complementing group 1 gene and pancreatic cancer risk: a systematic review with meta-analysis. Pathol Oncol Res. 2017.

38. Wang Z, Wu N. Association between XRCC1 and ERCC2 gene polymorphisms and development of osteosarcoma. Int J Clin Exp Pathol. 2016;9(1):223-229.

39. Pérez-Ramírez C, Cañadas-Garre M, Alnatsha A, et al. Pharmacogenetics of platinum-based chemotherapy: impact of DNA repair and folate metabolism gene polymorphisms on prognosis of non-small cell lung cancer patients. Pharmacogenomics J. 2018.
Cancer Management and Research

\section{Publish your work in this journal}

Cancer Management and Research is an international, peer-reviewed open access journal focusing on cancer research and the optimal use of preventative and integrated treatment interventions to achieve improved outcomes, enhanced survival and quality of life for the cancer patient. The manuscript management system is completely online and includes
Dovepress

a very quick and fair peer-review system, which is all easy to use. Visit $\mathrm{http}: / /$ www.dovepress.com/testimonials.php to read real quotes from published authors. 